\title{
A Cross Layer Time Slot Reservation Protocol for Wireless Networks
}

\author{
Bih-Hwang Lee, Chi-Ming Wong, and Hung-Chi Chien
}

\begin{abstract}
The function of medium access control (MAC) protocol and the capacity of physical layer sufficiently affect the system performance in the wireless networks. In this research, we propose a MAC protocol based on direct sequence code division multiple access (DS-CDMA) for the wireless networks to guarantee quality-of-service (QoS) which depend on physical layer information, named the cross layer time slot reservation (CLTSR) protocol. A channel is divided into control and data channels to transmit control and data packets in the corresponding control and data frames, respectively. The data frame is further subdivided into several time slots; each time slot can transmit different traffic types such as constant bit rate (CBR), variable bit rate (VBR), and available bit rate (ABR). Each station maintains the available spreading code (ASC) table to understand which time slots and spreading codes have been reserved. CLTSR will allocate time slots and the spreading code for the data frame by using the fixed time slot allocation (FTSA) or the mixed time slot allocation (MTSA). The QoS can be guaranteed by providing the reservation of time slots and the spreading code. A Markov model is applied to analyze the CLTSR DS-CDMA system; the analytical and simulation results show that the proposed CLTSR performs has been improved.
\end{abstract}

Keywords: medium access control (MAC), direct sequence code division multiple access (DS-CDMA), cross layer, quality-of-service (QoS).

\section{Introduction}

Wireless local area network (WLAN) has been widely used recently, which uses IEEE $802.11 \mathrm{x}$ protocol to coordinate all stations. Each station contends with each other by using the carrier sense multiple access with collision avoidance (CSMA/CA) to access the channel [1],[2]. CSMA/CA uses backoff algorithm to avoid collision and reduce the collision probability in the wireless environment. Several backoff algorithms have been proposed to improve system performance [3],[4], however, the performance is still limited by the physical capacity due to the use of single shared channel. Therefore, it is necessary to improve the cross layer functions between the physical layer and MAC layer.

For many years, some researches regarding the random access networking based on direct sequence code division multiple access (DS-CDMA) are studied [5]-[7], because multiple access interference (MAI) may be possibly suppressed in MAC layer. Generally in the controlled access CDMA (CA-CDMA) system, there exist control and data channels separately [8]. In the control channel, each station uses the signals of request-to-send (RTS) and clear-to-send (CTS) to contend the channel; and 
it also detects the degree of MAI to suitably adjust the transmitting power. However, this method needs more computational times and can not guarantee quality of service (QoS) in wireless network. Some MAC layer designs, such as the modified multicarrier CDMA based on IEEE 802.11a [9], CDMA based ad hoc network [10] and adaptive acquisition collision avoidance multiple access (AACAMA) [11], just give the way to get spreading codes and cannot limit the number of the used spreading codes to suppress MAI. They also can not guarantee QoS in wireless network. In the distributed channel allocation protocol (DCAP) [12], each frame is divided into several time slots; each node contends to get a pair of time slot-spreading code (TC) to transmit data. Similarly, it cannot limit the number of the used spreading codes and the allocation of time-slots, hence system performance will be degraded especially while the allocating algorithm doesn't provide the different maximum numbers of spreading codes for the different traffic types such as constant bit rate (CBR), variable bit rate (VBR) and available bit rate (ABR). Furthermore, both RTS and CTS in DCAP contain all TC pairs such that their packet sizes become longer, hence system performance will be degraded in heavy traffic.

In this paper, we use the DS-CDMA technology in physical layer and propose a cross layer time slot reservation (CLTSR) protocol in MAC layer for wireless system. CLTSR also divides the channel into control channel and data channel; each station uses the modified RTS (MRTS) and modified CTS (MCTS), data slot request (DSR) and short ACK (SACK) to reserve time slots and spreading code in the data frame by four way hand shaking (MRTS-MCTS-DSR-SACK). CLTSR supports the maximum numbers of the spreading codes for different traffic types to suppress MAI. All reserved time slots and spreading codes are put in DSR such that both MRTS and MCTS can keep shorter sizes. We provide two kinds of time slot allocation algorithm in MAC layer: fixed time slot allocation (FTSA) and mixed time slot allocation (MTSA) to allocate time slots and spreading codes depending on the required bandwidth for each traffic type, therefore the guaranteed QoS and the improved system performance can be achieved.

Next section will describe the proposed CLTSR based on DS-CDMA technology in wireless ad hoc network. Section III will do the theoretical analysis using the finite state Markov chain, and section IV will simulate the system operation and then show that the result. Finally, the conclusion is drawn in section V.

\section{Description of the Proposed CLTSR}

To design CLTSR, two frame structures for control channel and data channel are used and shown in Fig. 1. Data can be transmitted in the $\mathrm{k}^{\text {th }}$ data frame according to the competitive result in the $(\mathrm{k}-1)^{\mathrm{th}}$ control frame. A data frame is subdivided into $\mathrm{n}$ time slots as shown in Fig. 2, which can support different traffic types such as CBR, VBR and ABR. In the DS-CDMA system, the maximum number of channels can be simultaneously used to transmit data for CBR, VBR and ABR depending on the different tolerable bit error rate $\left(\mathrm{BER}, \mathrm{P}_{\mathrm{e}}\right)$ [13], which is assumed that BPSK modulation scheme is used in the additive white Gaussian noise (AWGN) channel as shown in Eq. (1) [14],

$$
\mathrm{P}_{\mathrm{e}}=Q(\sqrt{3 N / K-1})
$$


where $\mathrm{N}$ is the spreading factor, and $\mathrm{K}$ is the maximum number of channels used to transmit simultaneously, and $\mathrm{Q}(\mathrm{x})$ is the complementary error function. We assume the maximum sets of spreading codes to transmit data for CBR, VBR and ABR are $41,17,11$ corresponding to the bit error rate of $10^{-3}, 10^{-6}, 10^{-9}$, respectively, if the spreading factor is 128 .

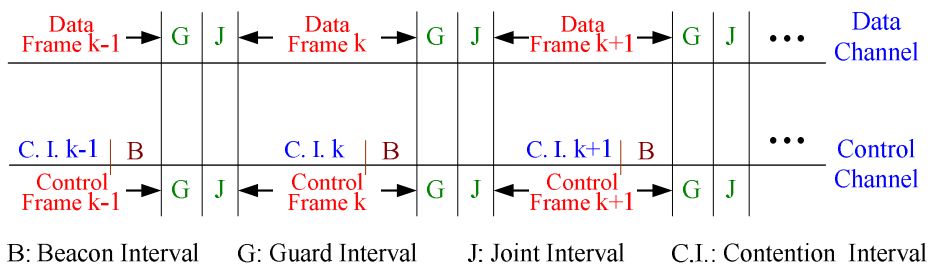

Fig. 1. The structure of control frame and data frame

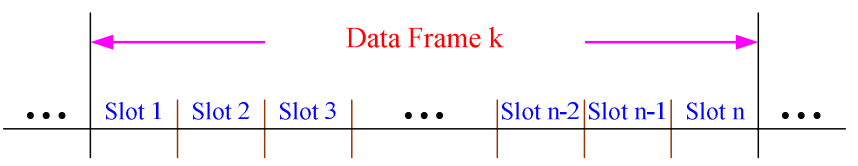

Fig. 2. The format of data frame

A control frame is divided into contention interval and beacon interval. The beacon interval is used to maintain system synchronization, while the contention interval is used to get the permission to send data in the next data frame. The contention is done by CSMA/CA with four-way hand shaking (MRTS-MCTS-DSR-SACK) procedure, where the formats of the corresponding control packets are shown in Fig. 3.

\begin{tabular}{|l|c|c|c|c|c|c|}
\hline $\begin{array}{c}\text { Frame } \\
\text { Control }\end{array}$ & RA & TA & $\begin{array}{c}\text { Traffic } \\
\text { Type }\end{array}$ & SCI & Reserved & FCS \\
\hline
\end{tabular}

(a) The format of MRTS

\begin{tabular}{|l|l|l|l|l|l|}
\hline $\begin{array}{c}\text { Frame } \\
\text { Control }\end{array}$ & RA & $\begin{array}{c}\text { Traffic } \\
\text { Type }\end{array}$ & SCI & Reserved & FCS \\
\hline
\end{tabular}

(b) The format of MCTS

\begin{tabular}{|l|c|c|c|c|c|c|c|c|c|}
\hline $\begin{array}{c}\text { Frame } \\
\text { Control }\end{array}$ & RA & TA & SNR & $\begin{array}{c}1_{\text {st }} \text { slot } \\
\text { Number }\end{array}$ & $\begin{array}{c}2_{\text {nd }} \text { slot } \\
\text { Number }\end{array}$ & $\cdots$ & $\begin{array}{c}(\mathrm{m}-1)_{\text {th }} \text { slot } \\
\text { Number }\end{array}$ & $\begin{array}{c}\mathrm{m}_{\text {th }} \text { slot } \\
\text { Number }\end{array}$ & FCS \\
\hline
\end{tabular}

(c) The format of DSR

\begin{tabular}{|l|l|l|}
\hline $\begin{array}{c}\text { Frame } \\
\text { Control }\end{array}$ & RA & FCS \\
\hline
\end{tabular}

(d) The format of SACK

Fig. 3. The format of control packets 
The field of traffic type may consist of two bits to represent three traffic types. The spreading code index (SCI) is indicated in the field of "SCI", which may has six bits to indicate at most 41 sets of spreading codes. The fields of "RA" and "TA" represent the MAC addresses for receiver and transmitter respectively. The field of the slot number reservation (SNR) is used to reserve the number of time slots to be transmitted in the data frame, whose content depends on the transmission bandwidth requirement. Let $N_{s}$ be the number of time slots to be reserved in the data frame and to be obtained by Eq. (2).

$$
N_{s}=\left\lceil\frac{r_{b}}{r_{f} * b_{s}}\right\rceil
$$

where $r_{b}$ is the required transmission bandwidth in bit per second (bps); $r_{f}$ is the number of data frames per second; and $b_{s}$ is the transmitted bits in each time slot. The field of "Slot Number" represents the reserved time slots in the data frame, and the amount of "Slot Number" is the same as SNR. Thus, the size of DSR packet depends on how many time slots are needed. The functions of "Frame Control" and "FCS" are the same as the IEEE 802.11 standard [15].

Next, we introduce the procedure of CLTSR to access channel through the control packets.

Step 1: Use allocation algorithm to reserve time slots and spreading codes for transmission in the next data frame.

Step 2: Use the four-way handshaking procedure to contend and get the permission of time slots and spreading codes.

Step 3: Monitor the DSR packets from other stations to check the confliction. It must run time slot allocation algorithm again when the content of its DSR packet conflicts with other stations, and go to step 2 .

Step 4: Use those time slots and spreading codes to transmit frames until the transmission finished, then releases the time slots and spreading codes.

Table 1. Initialization of ASC Table

\begin{tabular}{|c|c|c|c|c|c|c|}
\hline & Slot 1 & Slot 2 & $\ldots$ & Slot n-1 & Slot $\mathrm{n}$ \\
\hline \multicolumn{2}{|c|}{ Traffic types } & 0 & 0 & $\ldots$ & 0 & 0 \\
\hline \multirow{8}{*}{ SCI } & 1 & $\operatorname{TCI}(1,1)$ & TCI $(1,2)$ & $\ldots$ & $\mathrm{TCI}(1, \mathrm{n}-1)$ & $\mathrm{TCI}(1, \mathrm{n})$ \\
\hline & 2 & $\mathrm{TCI}(2,1)$ & $\operatorname{TCI}(2,2)$ & $\ldots$ & $\mathrm{TCI}(2, \mathrm{n}-1)$ & $\operatorname{TCI}(2, \mathrm{n})$ \\
\hline & & : & $:$ & $\ldots$ & : & : \\
\hline & 11 & $\operatorname{TCI}(11,1)$ & $\operatorname{TCI}(11,2)$ & $\ldots$ & $\mathrm{TCI}(11, \mathrm{n}-1)$ & $\mathrm{TCI}(11, \mathrm{n})$ \\
\hline & & : & : & $\ldots$ & : & : \\
\hline & 17 & $\operatorname{TCI}(17,1)$ & $\operatorname{TCI}(17,2)$ & $\ldots$ & $\operatorname{TCI}(17, \mathrm{n}-1)$ & $\operatorname{TCI}(17, \mathrm{n})$ \\
\hline & & : & : & $\ldots$ & : & : \\
\hline & 41 & $\mathrm{TCI}(41,1)$ & $\mathrm{TCI}(41,2)$ & $\ldots$ & $\mathrm{TCI}(41, \mathrm{n}-1)$ & $\mathrm{TCI}(41, \mathrm{n})$ \\
\hline
\end{tabular}


Each station in the system must cooperate each other well by some distributed resource allocation algorithms because there has no base station. FTSA and MTSA allocate time slots and spreading codes according to the available spreading code (ASC) table in each station, where the initialization of ASC table is shown in table 1. A time slot spreading code index (TCI) pair is a pair of time slot and spreading code in the ASC table, while the marked TCI pair in ASC table means that this TCI pair has been reserved already. The traffic type in each time slot is decided by the first station of using it, which is set to 0,1 , or 2 for CBR, VBR, and ABR, respectively. The FTSA algorithm is described as follows.

Step 1: Search from ASC table to find all time slots having the same traffic type and available TCI pairs relative to those time slots.

Step 2: Allocate number of TCI pairs which obtained by Eq. (2) from those available TCI pairs with a spreading code in best fit.

Step 3: Try to find enough free time slots and set them to this traffic type if the TCI pairs is not enough.

Step 4: Go to step 1 if there are still no enough free time slots, then the station enters blocking state.

The MTSA algorithm is obtained by modifying the FTSA algorithm and described as follows.

Step 1: The same as step 1 in FTSA.

Step 2: The same as step 2 in FTSA.

Step 3: The same as step 3 in FTSA.

Step 4: Try to find TCI pairs of VBR traffic to be used for CBR traffic.

Step 5: The same as step 4 in FTSA.

\section{Theoretical Analysis}

This section analyzes the proposed CLTSR with FTSA because MTSA is similar to FTSA. We assume that the arrival traffics are the Poisson process with the mean arrival rates $\lambda_{C}, \lambda_{V}$, and $\lambda_{A}$, for the traffic types CBR, VBR and ABR, respectively. The service processes are also assumed to be exponentially distributed with the mean service rates $\mu_{C}, \mu_{V}$, and $\mu_{A}$, for the traffic types CBR, VBR and ABR, respectively. Throughput can be obtained depending on how many TCI pairs are occupied by each traffic type. The TCI pair occupied by any traffic can be modeled by Markov chain. We assume that there are $n$ time slots in each data frame, and the maximum number of TCI pairs can be occupied by CBR, VBR, and ABR are $n_{C}, n_{V}$, and $n_{A}$, respectively, but Eq.(3) must be satisfied.

$$
\left\lceil\frac{n_{C}}{41}\right\rceil+\left\lceil\frac{n_{V}}{17}\right\rceil+\left\lceil\frac{n_{A}}{11}\right\rceil \leq n
$$




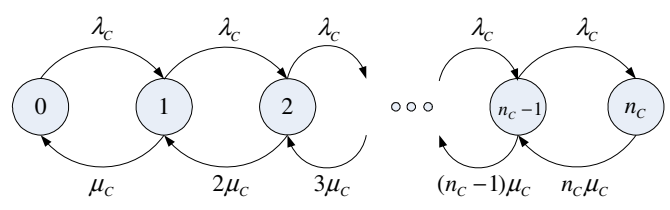

Fig. 5. State transition diagram of the CBR traffic

The number of TCI pair occupied by CBR traffic can be modeled by Markov chain with $n_{C}$ states and its state transition diagram is shown as Fig. 5. We assume that only one TCI pair can be occupied whenever the CBR type traffic arrives. The state transition of the Markov chain can be characterized by a generator matrix. Let us denote $\mathbf{Q}_{C}$ be the generator matrix for CBR traffic shown as Eq. (4), while $\mathbf{P}_{C}$ represents the steady state probability vector for CBR traffic, where $\mathbf{P}_{\mathrm{C}}=\left[\begin{array}{lllll}p_{C 0} & p_{C 1} & p_{C 2} & \cdots & p_{C n c}\end{array}\right]$. The element of $\mathbf{P}_{\mathrm{C}}$ represents the steady state probability of each state in Fig. 5, which can be obtained by solving the balance equation shown as Eq. (5).

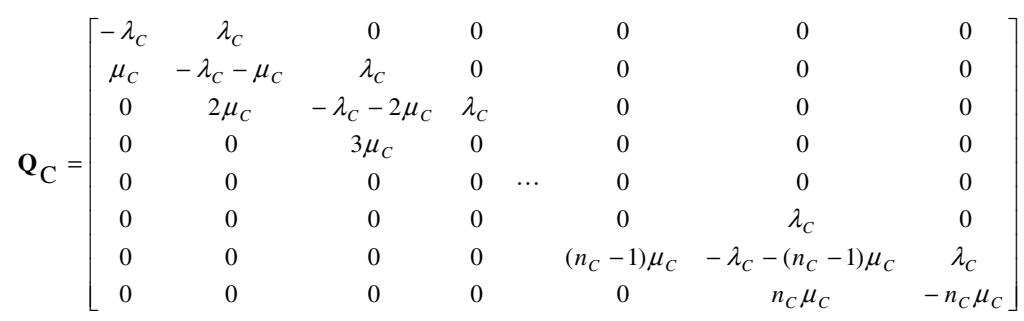

Then, we have $\mathbf{P}_{\mathrm{C}} \mathbf{Q}_{\mathrm{C}}=\mathbf{0}$

Similarly, the generator matrices for VBR and ABR traffics can also be obtained.

The TCI pairs occupied by VBR or ABR traffics are random variables, because the required bandwidths for $\mathrm{VBR}$ and $\mathrm{ABR}$ traffics are also random variables; therefore, we should transform the distribution of bandwidth requirement to the distribution of TCI pairs' requirement for VBR and ABR traffics. Let us denote $f_{V R}(r)$ be the probability density function of the bandwidth requirement for VBR traffic with boundary between $R_{\min }$ and $R_{\max }$ (bps). Let $m_{l}$ and $m_{u}$ represent the minimum and maximum number of TCI pairs occupied by VBR traffic and have the relationship with $m_{u}>m_{l}>1$, where $m_{l}=\frac{R_{\min }}{B_{s}}$ and $m_{u}=\frac{R_{\max }}{B_{s}}$ if $B_{s}$ is the transmission rate of a TCI pair in data frames per second. The probability distribution of the number of TCI pairs' requirement for VBR traffic can be represented by a vector $\mathbf{b}_{V}=\left[\begin{array}{lllll}b_{V 0} & b_{V 1} & b_{V 2} & \cdots & b_{V m}\end{array}\right]$, where $m=m_{u}-m_{l}$. The element $b_{V k}(k=0,1, \ldots, m)$ in vector $\mathbf{b}_{V}$ represents the probability that there are $\left(m_{l}+k\right)$ TCI pairs required by VBR traffic and can be calculated by Eq. (5). 


$$
b_{V k}= \begin{cases}\int_{0}^{R_{\min }} f_{V R}(r) d r, & \text { for } k=0 \\ \int_{\left(m_{l}+k-1\right) B_{s}}^{\left(m_{l}+k\right) B_{s}} f_{V R}(r) d r, & \text { for } k=1,2, \ldots, m-1 \\ \int_{\left(m_{u}-1\right) B_{s}}^{\infty} f_{V R}(r) d r, & \text { for } k=m\end{cases}
$$

Note that $\sum_{k=0}^{m} b_{V k}=1$. Actually, CBR traffic can be regarded as a special case of VBR traffic, as $m_{l}=m_{u}=1$ and $m=0$. Therefore, the arrival and departure rates for VBR traffic from a state can be obtained by the vectors $\lambda_{\mathrm{V}} \mathbf{b}_{V}$ and $\mu_{\mathrm{V}} \mathbf{b}_{V}$, respectively.

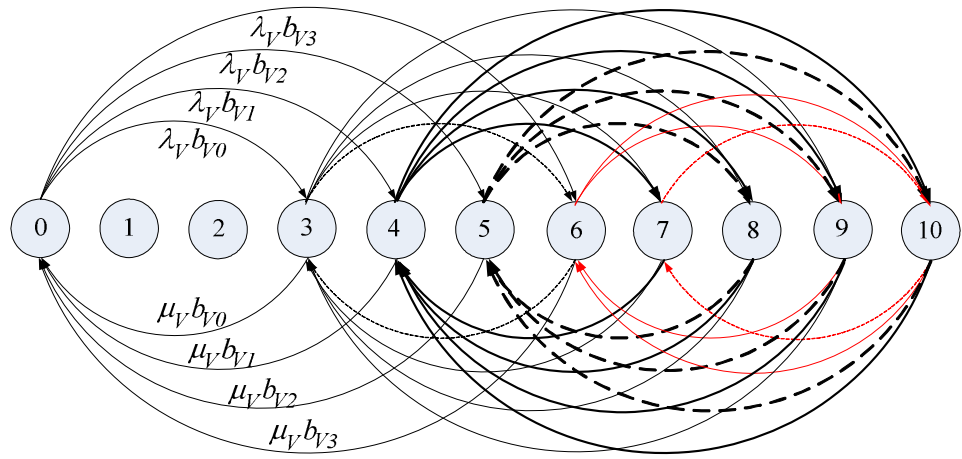

Fig. 6. Example state transition diagram of VBR traffic

For example, if $m_{l}$ and $m_{u}$ equal 3 and 6, respectively, then $m$ equals 3 . If $n_{V}$ equals 10, the state transition diagram is shown as Fig. 6 and the reduced generator matrix $\mathbf{Q}_{\mathrm{V}}$ has the dimension of $9 \times 9$ shown as Eq. (7). In Fig. 6, it is obvious that the states 1 and 2 will never be reached so that their probabilities are zero. The transition probabilities $p_{01}, p_{02}, \ldots, p_{0\left(m_{l}-1\right)}$ and $p_{10}, p_{20}, \ldots, p_{\left(m_{l}-1\right) 0}$ will be zero, because the minimum requirement of TCI pairs is $m_{l}>1$. Some states may have more than one possible transitions for $m \geq m_{l}$. For example, the $6^{\text {th }}$ state may be transited from the initial state with probability $\lambda_{v} b_{v 3}$ or from the $3^{\text {rd }}$ state with probability $\lambda_{v} b_{v 0}$, while the transition rate from the $9^{\text {th }}$ state to the $6^{\text {th }}$ state can be obtained to be $3 \mu_{v} b_{v 0}^{2}+2 \mu_{v} b_{v 0} b_{v 3}$.

$$
\mathbf{Q}_{V}=\left[\begin{array}{ccccccccc}
q_{v 00} & \lambda_{V} b_{V 0} & \lambda_{V} b_{V 1} & \lambda_{V} b_{V 2} & \lambda_{V} b_{V 3} & 0 & 0 & 0 & 0 \\
\mu_{V} b_{V 0} & q_{v 11} & 0 & 0 & \lambda_{v} b_{V 0} & \lambda_{v} b_{V 1} & \lambda_{V} b_{V 2} & \lambda_{v} b_{V 3} & 0 \\
\mu_{V} b_{V 1} & 0 & q_{v 22} & 0 & 0 & \lambda_{v} b_{V 0} & \lambda_{V} b_{V 1} & \lambda_{V} b_{V 2} & \lambda_{V} b_{V 3} \\
\mu_{V} b_{V 2} & 0 & 0 & q_{v 33} & 0 & 0 & \lambda_{V} b_{V 0} & \lambda_{V} b_{V 1} & \lambda_{V} b_{V 2} \\
\mu_{V} b_{V 3} & 2 \mu_{V} b_{V 0} & 0 & 0 & q_{v 44} & 0 & 0 & \lambda_{v} b_{V 0} & \lambda_{V} b_{V 1} \\
0 & 2 \mu_{V} b_{V 1} & 2 \mu_{V} b_{V 0} & 0 & 0 & q_{v 55} & 0 & 0 & \lambda_{v} b_{V 0} \\
0 & 2 \mu_{V} b_{V 2} & 2 \mu_{V} b_{V 1} & 2 \mu_{V} b_{V 0} & 0 & 0 & q_{v 66} & 0 & 0 \\
0 & 2 \mu_{V} b_{V 3} & 2 \mu_{V} b_{V 2} & 2 \mu_{V} b_{V 1} & 3 \mu_{V} b_{V 0}^{2}+2 \mu_{V} b_{V 0} b_{V 3} & 0 & 0 & q_{v 77} & 0 \\
0 & 0 & 2 \mu_{V} b_{V 3} & 2 \mu_{V} b_{V 2} & 3 \mu_{V} b_{V 1} b_{V 0}+2 \mu_{V} b_{V 1} b_{V 3} & 3 \mu_{V} b_{V 0} & 0 & 0 & q_{v 88}
\end{array}\right]
$$


Similarly, the reduced generator matrix for ABR traffic $\mathbf{Q}_{\mathrm{A}}$ can also be obtained by substituting $\lambda_{\mathrm{A}}$ and $\mu_{\mathrm{A}}$ for $\lambda_{\mathrm{V}}$ and $\mu_{\mathrm{V}}$, respectively. The steady state probability vector for $\mathrm{ABR}$ traffic $\mathbf{p}_{\mathrm{A}}$ can be obtained by solving the balance equation shown as Eq. (8).

$$
\mathbf{p}_{A} \mathbf{Q}_{A}=\mathbf{0}
$$

\section{Simulation Results}

In this section, we simulate the proposed CLTSR system by using Visual C++ program and compare with the theoretical analysis. Let us assume that data channel has the bandwidth of $10.6 \mathrm{Mbps}$; each time slot has the length 2 kbits (about $0.1887 \mathrm{~ms}$ ); each data frame has 164 time slots (about 30.94ms); and there yields about 32 data frames per second. Let us also assume that the arrival traffics of CBR, VBR and ABR occupy $19 \%, 4 \%$ and $77 \%$, respectively. The average bandwidth requirement for $\mathrm{CBR}$, VBR and $\mathrm{ABR}$ traffics are 64, 512, and $256 \mathrm{kbps}$, respectively. Similarly, the mean access duration for CBR, VBR and ABR traffics are 5, 10 and 1.56 minutes, respectively. Throughput is calculated by $\left(N_{p k t} \times l\right) / T_{\text {sim }}$, where $l, N_{p k t}$ and $T_{\text {sim }}$ are the packet length, the total number of the successful transmitted packets and the simulation time. The blocking probability is obtained by $N_{b} /\left(N_{b}+N_{s}\right)$ where $N_{b}$ and $N_{s}$ are the number of blocking and success packets, respectively.

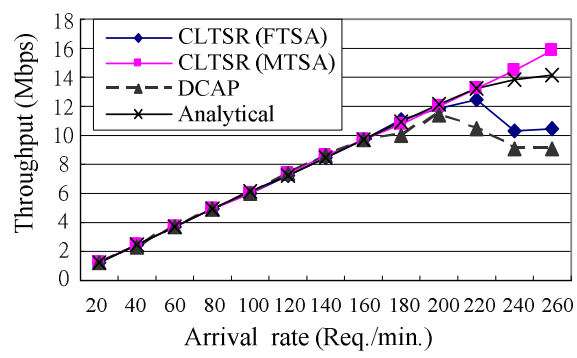

(a) CBR traffic



(c) ABR traffic



(b) VBR traffic

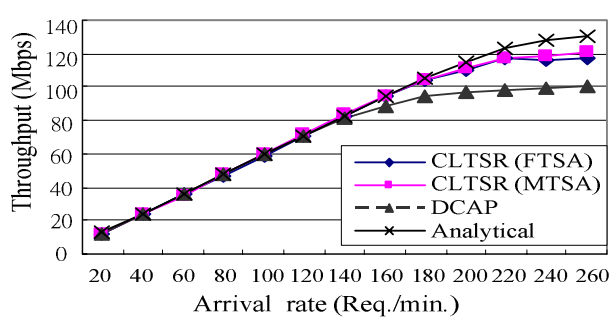

(d) Combining CBR, VBR, and ABR traffics

Fig. 7. Throughput versus arrival rate for CLTSR, DCAP and analytical solutions 
Fig. 7 shows the simulated throughput for FTSA/MTSA in CLTSR, DCAP and theoretical analysis obtained from the Markov model above. We see that the proposed Markov model fits the simulation results very well except arrival traffic higher than 220 (Req./min.). Although the CBR traffic occupy $19 \%$ of arrival traffic, the allowable 41 sets of spreading codes in a time slot and 64Kbps constant bandwidth requirement will make it occupying time slot in the data frame slower than ABR traffic in almost no available time slots can be used. And it also means that when the arrival traffic is heavy (over 220 (Req./min.)), the throughput of CBR traffic will lower than theoretical analysis. But once the MTSA is used, we can see the throughput of CBR traffic even higher than theoretical analysis, as shown in Fig. 7 (b). One way to make throughput always near the theoretical analysis is expanded time slot or channel capacity in the data channel. Of course, due to the DCAP hasn't good time slot allocation algorithm to allocate time slot, it will have lower performance than CLTSR system. Similarly, the VBR traffic has same situation as the CBR traffic, as shown in Fig. 7 (c). And we can see the throughput of VBR using MTSA is same as using FTSA. So, the MTSA never affect the throughput in VBR traffic but enhance the throughput in CBR traffic. In the Fig. 7 (d), the FTSA, MTSA and theoretical analysis are almost same everywhere in the ABR traffic. Fewer sets of spreading codes, smaller access duration and higher occupation of arrival traffic make ABR traffic has more stable situation.

\section{Conclusions}

We have proposed the CLTSR for DS-CDMA based wireless network. The special reservation protocol which combines the function of DS-CDMA in physical layer and time slots/spreading code allocation in MAC layer not only improve system performance but also provide the guarantee of QoS. It is shown that the Markov chain models are accurate in predicting the behavior of CLTSR under the assumption that the arrival traffic has Poisson distribution. The MTSA is suggested to use because the performance of VBR and ABR traffic are almost same in both FTSA and MTSA, while the MTSA has better performance in CBR traffic. This is a special MAC protocol design dependent on the capacity of physical layer. Due to the advance multiple access and channel estimation technology in wireless communication networking, we need a better MAC protocol to use them. Then, the performance with respect to the entire system can have the best situation. This is the motive that we begin to design the CLTSR.

\section{References}

1. Bianchi, Y.G.: Performance Analysis of the IEEE 802.11 Distributed Coordination Function. IEEE Journal on Selected Areas in Communications 18(3), 535-547 (2000)

2. Robinson, J.W., Randhawa, T.S.: Saturation throughput analysis of IEEE 802.11e enhanced distributed coordination function. IEEE Journal on Selected Areas in Communications 22(5), 917-928 (2004)

3. Kwon, Y., Fang, Y., Latchman, H.: Design of MAC Protocol With Fast Collsion Resolution for Wireless Local Area Networks. IEEE Trans. Wireless Commun. 3(3), 793-807 (2004) 
4. Haas, Z.J., Deng, J.: On Optimizing the Backoff Interval for Random Access Schemes. IEEE Trans. Commun. 51(12), 2081-2090 (2003)

5. Raychaudhuri, D.: Performance analysis of random access packet-switched code division multiple access systems. IEEE Trans. Commun. COM-29(6), 895-901 (1981)

6. Morrow, R., Lehnert, J.: Packet throughput in slotted ALOHA DS/SSMA radio systems with random signature sequences. IEEE Trans. Commun. 40(7), 1223-1230 (1992)

7. Sato, T., Okada, H., Yamazato, T.: Throughput Analysis of DS/SSMA Unslotted ALOHA System with Fixed Packed Length. IEEE Journal on Selected Areas in Communications 14(4), 750-756 (1996)

8. Muqattash, A., Krunz, M.: CDMA-Based MAC Protocol for Wireless Ad Hoc Networks. In: Proceedings of the $4 \mathrm{ACM}$ international symposium on Mobile ad hoc networking \& computing (July 2003)

9. Orfanos, G., Habetha, J., Liu, L.: MC-CDMA Based IEEE 802.11 Wireless LAN. In: IEEE Computer Society's 12th Annual International Symposium, pp. 400-405 (October 2004)

10. Fantacci, R., Ferri, A., Tarchi, D.: A MAC technique for CDMA based Ad-Hoc networks. In: IEEE Wireless Communications and Networking Conference, vol. 1, pp. 645-650 (March 2005)

11. Qiang, G., Liu, Z., Ishihara, S., Mizuno, T.: CDMA-based Carrier Sense Multiple Access Protocol for Wireless LAN. In: IEEE Vehicular Technology Conference 53rd, vol. 2 (May 2001)

12. Yang, H., Kim, K.: Multimedia Ad Hoc Wireless LANs with Distributed Channel Allocation Based on OFDM-CDMA. IEICE Trans. Commun. E86-B(7) (2003)

13. Akyildiz, I.F., Levine, D.A., Joe, I.: A slotted CDMA protocol with BER scheduling for wireless multimedia networks. IEEE/ACM Trans. Networking 7(2), 146-158 (1999)

14. Rappaport, T.S.: Wireless Communications PRINCIPLES AND PRACTICE, 2nd edn. pp. 621-650. Prentice Hall, Englewood Cliffs (2002)

15. Wireless LAN Medium Access Control (MAC) and Physical Layer (PHY) specifications, ANSI/IEEE std. 802.11 